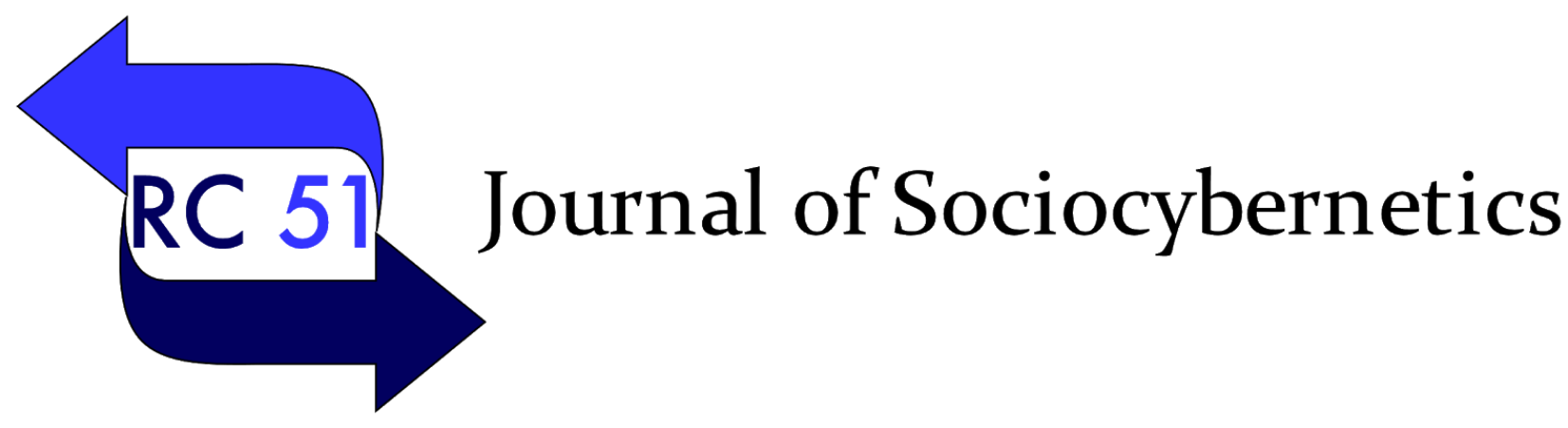

Journal of Sociocybernetics ISSN 1607-8667

\title{
Questioning the algorithmic transparency of location-based platforms
}

\author{
Lorenza Parisi, Giovanni Andrea Parente
}

Link Campus University

\begin{abstract}
In the platform society, algorithms are seen as 'black boxes' (Pasquale, 2015) and users have only a vague understanding of the criteria they adopt to select and filter information. Location-based platforms' algorithms influence the visibility of different points of interest (POI), thus shaping user interaction with venues and places. This paper will adapt the Diakopoulos and Koliska model (2017), present a new framework for analysing the algorithmic transparency of location-based platforms and apply it to three popular location-based platforms (Google Maps, Tripadvisor and Instagram). The research questions are the following: RQ1) How do location-based platforms communicate algorithmic transparency?; $R Q_{2}$ ) Which are the most relevant dimensions they take into consideration (data, model, inference and interface)?; $R Q_{3}$ ) How do platforms communicate transparency toward different targets (i.e., consumers and suppliers)? Following Rader, Cotter and Cho (2018), it is expected that location-based platforms are less transparent about the data they manage and model they use and slightly more transparent about the inferences (ranking, suggestions and ads). Moreover, we expect location-based platforms to be more transparent toward suppliers rather than consumers. This paper will assess how Google Maps, Tripadvisor and Instagram disclose algorithmic transparency as it emerges from the analysis of 'extant' online data that has been officially released (policies, guidelines and tutorials) and from the analysis of the platforms' mobile interfaces. The analysis reveals that platforms are less transparent about the data they manage and model they use and more transparent, solely toward suppliers, about the inferences they propose. Moreover, location-based platforms are more transparent toward suppliers rather than consumers; indeed, commercial interests favour the algorithmic transparency of location-based content.
\end{abstract}

url: https://doi.org/10.26754/ojs_jos/jos.202013929

\section{Introduction}

In the platform society (van Dijck, Poell, \& de Waal, 2018) proprietary algorithms filter, arrange and suggest information. Traditionally, experts and institutions played key roles in selecting information, but nowadays "online platforms replace experts-based selection with user-driven and algorithm-driven selection” (ibid., p. 40). Algorithms are perceived as 'black boxes' (Pasquale, 2015) and users (whether they are consumers or suppliers) have only a vague understanding of the ranking criteria they adopt. Indeed, algorithms are non-neutral, and search results and recommendations are influenced by several variables: user preferences; access to network locations; previously performed queries; other users' interactions with specific content. While algorithms are generally perceived to

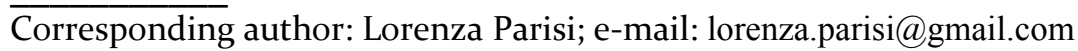


be innately 'democratic' (Gillespie, 2017), they in fact follow the techno-commercial strategies of their owners and arrange information accordingly. Within this scenario, location-based platforms represent an interesting case study to investigate how algorithms shape information-seeking and, therefore, the visibility of points of interest (POI) and activities taking place in the (urban) space (Parisi, 2015).

This paper will investigate the transparency of location-based algorithms and is organised as follows: The literature review refers to the critical algorithm studies approach and points out the importance of investigating algorithms' transparency of location-based media. The analysis focuses on three popular location-based platforms (Google Maps, Tripadvisor and Instagram) used for touristic information (e.g., searching for food and drink facilities). Despite being aware that these platforms are different in terms of goals, users and owners, the aim of this paper is to investigate a variety of apps used to search for location-based information in the context of touristic mobility so as to discover common trends and differences in the way different platforms address algorithmic transparency. This paper adapts the model Diakopoulos and Koliska (2017) developed for analysing algorithmic transparency in news media and develops a new model for analysing how location-based platforms disclose information around the algorithms they employ. We consulted several official documents addressed to different users (consumers and suppliers) and we performed several queries on the apps. The research investigates how location-based platform owners disclose algorithmic transparency in relation to 4 layers (data, model, inference and interface).

The theoretical framework has benefited from a productive dialogue with the Actor-Network Theory approach (Law \& Hassard, 1999). Indeed, the analysis of the interactions between location-based platforms and users fits perfectly with a systemic approach which analyses human and non-human actors in a symmetrical way, incorporating into a wider network all the social processes influencing such interactions. Indeed, non-humans (e.g., algorithms) can also learn from interaction, thus creating alliances and relationships among human, social and technological elements. Considering human and non-human actors as part of the same network (Law, 2007) means that digital algorithms can be seen as socio-digital assemblages and actors per se (Introna, 2016). This vision resonates with Pickering's (1995) invite to modify the idea of 'social' which sees the domination of human action. Indeed, human beings do not always assume a central role within a social system, and they are part of an ongoing dialectic between human and non-human agencies.

\section{Questioning algorithmic transparency of location-based platforms}

Scholars following a critical algorithm studies approach point out that algorithms play an important cultural role in shaping the boundaries of what is knowable. However, most users do not question the 'hidden' logics behind the proprietary algorithms driving suggestions within different apps (e.g., dating apps, apps for ordering food or booking a hotel, planning a trip or finding the nearest place to eat). On the contrary, users seem to appreciate hyper-simplified search engines that reduce the number of occurrences and simplify the decision-making process. According to van Dijck, Poell and de Waal (2018), three main types of selection are at play in platform dynamics: personalisation, reputation and trends, and moderation. This implies that user agency is diminished, given that each platform provides them with limited options, according to filters (preferences), parameters and other logics (e.g., tracking of user activities), that are anything but transparent.

As Cardon (2016) explained, digital algorithms can assess different phenomena: popularity, authority, reputation and personalisation. Moreover, algorithmic logic is mostly 'conservative' and aims to maximise the time users spend on the platform: algorithms tend to provide us with 
personalised information that matches with our pre-existing interests, thus reinforcing 'filter bubbles' (Pariser, 2011). In the last few years there has been a growing interest in investigating the values embedded in digital algorithms, the ideology they propose and the non-neutral criteria that determine the visibility of information in search engine result pages and social media user feeds. Following this approach, the focus of this paper is not on describing the technicalities around a specific algorithm but rather exploring the algorithmic transparency of location-based platforms by looking at documentation officially released by the platforms and from analysis of the user interfaces.

Generally speaking, new Web 2.0 apps, and location-based platforms specifically, are often perceived in their initial stage as expressions of participatory culture (Jenkins, 2008) and innately grassroot as they are based on user-generated content (UGC). For example, YouTube was created as a digital video repository for sharing videos among friends; it subsequently turned into a huge media company (Burgess, \& Green, 2009). As a result, mainstream online platforms are based on a "systematic collection, algorithmic processing, circulation, and monetization of user data" (van Dijck, Poell \& de Waal, 2018). Indeed, several tech corporations benefited from this scenario and now exploit content produced by the audience, record personal data and then sell it to advertising clients (Fuchs, 2017); this has led to a concentration of economic power in the hands of a few platform owners.

Platforms also benefit from the precision of data analysis tools, enabling highly effective user profiling (Nieborg \& Poell, 2018). This process is part of datafication (van Dijck, Poell \& de Waal, 2018), which allows platforms to transform into data different aspects of the world never quantified before (Mayer-Schönberger \& Cukier, 2013). As a result, platforms sell data about user behaviour to third parties with which they have commercial agreements and develop predictive analysis to create more tailored and personalised services. In fact, in order to increase their revenues, the algorithms of Internet-based companies, in conformity with business interests, prioritise information that already benefits from a preferential position in the network - "the rich get richer" phenomenon (Barabasi, 2004) - or that are already implementing digital marketing strategies. Indeed, platform capitalism is based on the exploitation of the labour of the users, selling user attention to advertising clients, so the more time users spend on a platform, the more profits tech companies generate. In relation to location-based platforms this means that some points of interest (POIs) and the related location-based data (photos, reviews, etc.) are more visible than others. In this paper, in order to observe how platforms and users deal with this inevitable prioritisation of content, we decided to circumscribe the analysis to a case study, focusing on location-based platforms used to access information about food and drink facilities.

Introna and Nissenbaum (2000) were among the first scholars investigating the topic of 'algorithmic transparency' and advocating transparency in relation to search engines. More specifically, in the context of digital news-making, scholars outlined that algorithms act as gatekeepers (Granka, 2010) and demanded guidelines to facilitate the disclosure of algorithmic systems (Diakopoulos \& Koliska, 2017). At the same time, other scholars warned against the risks of an excess of transparency that might lead to potential hacker attacks or search result manipulation (Laidlaw, 2008). Recently, European policy makers adopted the General Data Protection Regulation (GDPR, 2019) which introduced the "right to explanation" of decisions made by algorithms (art. 22), supporting the principle that the explanation of how an algorithm works has to be articulated and understood in such a way that it is clear (Goodman, 2016). Rader, Cotter and Cho (2018) observed that digital platform policies provide more explanation of suggestions and recommendations and pay 
less attention to providing explanations about data (sources of data and the use of personal information) or about the model (algorithmic decision-making system). In relation to the use of data, Obar and Oeldorf-Hirsch (2018) explain that individuals tend to ignore privacy policy (PP) and terms of service (TOS) when joining a new service. In an experimental study they created a fictitious social network service and discovered that users took an average of around one minute to read the policies, when it should have taken several (29-32 minutes for the PP and 15-17 minutes for the TOS). As they observed, information overload is a significative negative predictor of reading privacy policy and terms of service. Indeed, as the documentary Terms and conditions may apply (2013) demonstrated, long terms and conditions that employ long-winded and uppercase text look uninviting.

The topic of algorithmic transparency is also very important in relation to location-based platforms. Nowadays, almost all mobile apps, services and social network sites employ a location-based feature and urban dwellers increasingly rely on these platforms to get information about different places and services. As a result, these platforms shape our actual place experience the chances of visiting an attraction, trying a new restaurant, taking a specific bus - and the overall perception and reputation of specific locations. For example, according to several studies (Varkaris \& Neuhofer, 2017), the above mentioned platforms and use of social media affect a consumer's decision-making process, influencing both their search activities while they are on-the-go and their final decision to book a hotel.

This paper is part of a wider research project investigating the transparency of location-based algorithms with a focus on two main actors: platforms and users. Moreover, in relation to the users, we introduced an additional distinction between: 1) consumers (people using location-based platforms for personal needs); 2) suppliers, namely professionals or organisations using a platform to manage the visibility and reputation of a specific venue (e.g., restaurant owners using Tripadvisor). This paper constitutes the first research step and introduces a model to analyse the algorithmic transparency of location-based platforms. Then it focuses on the platform side and analyses how location-based platforms officially disclose information around the algorithms they employ. The next research step is underway and applies the aforementioned model to investigate how users (suppliers and consumers) perceive location-based platform transparency.

\section{Research Method}

The main research question is the following: How do location-based platforms disclose algorithmic transparency in relation to the four layers identified by the Diakopoulos and Koliska model (data, model, inference and interface)? Following Rader, Cotter and Cho (2018), it is expected that location-based platforms are less transparent about the data they manage and about their model and slightly more transparent about the criteria guiding the inferences they make (e.g., ranking, personalised suggestions and sponsored content). Moreover, it is expected that location-based platforms are more transparent toward suppliers than consumers.

People use a wide variety of location-based apps in their everyday life. This analysis focuses on three particularly popular yet different location-based platforms used to get information about food and drink facilities: Google Maps, Tripadvisor and Instagram. Rather than comparing the above-mentioned apps, this paper investigates how these platforms officially disclose information about location-based proprietary algorithms and how they influence the findability, visibility and relevance of POIs, in order to discover common trends and differences. Indeed, the chosen apps are very different in their scopes, users and ownership: Google Maps supports a generalist use, for 
getting information about different venues or checking traffic conditions; Tripadvisor is more specific and is mainly used to search for hotels, attractions, services and restaurants; Instagram indirectly shapes user perceptions of a place by showing images and videos related to events, venues and locations, thus influencing the chances of visiting a place. We executed five queries each day for one month (1oth May - 1oth June 2019) using the mobile interface of the above-mentioned apps. Queries simulated common tasks performed by users of the above mentioned apps: searching for a Point of interest (e.g. a specific restaurant) or an activity; looking at location-based results on the search engine results page and the related map; exploring related information about venues and places. Then the research analyses 'extant' online data (Salmons, 2015): ten official documents (privacy policy, terms of service, data policy and guidelines) and several tutorials and help pages (as they appeared on the 31 May 2019) addressed to Italian users (consumers and suppliers). The Appendix lists the full list of documents consulted ${ }^{1}$.

This paper adapts the Diakopoulos and Koliska model (2017) they applied to analyse algorithmic transparency in news media and presents a new analytical model for analysing the algorithmic transparency of location-based platforms. In relation to each layer, we verified the appropriateness of the factors described in the Diakopoulos and Koliska model and, if needed, we added new factors to better describe location-based algorithmic transparency. As table 1 shows, in the first layer, Data, we analyse the transparency in relation to the sources, use and storage of personal data. In the second layer, Model, transparency was investigated in relation to the input variables classifying a POI. In the third layer, Inference, transparency was examined in relation to the criteria informing results (e.g., ranking, personalised suggestions, paid placement, etc.). Finally, in the fourth layer, Interface, transparency was investigated in terms of how the interface, mostly based on a map, visualises location-based data (e.g., some POIs are more findable, visible and relevant than others).

\begin{tabular}{|c|c|c|}
\hline Layer & Factors & Definition \\
\hline \multirow[t]{5}{*}{ Data } & Database size & The overall size of the database. \\
\hline & Paid inclusion & The POI is paying a fee to be included in the database. \\
\hline & Personal data & $\begin{array}{l}\text { The app informs the user about which personal data it } \\
\text { collects about the user. }\end{array}$ \\
\hline & $\begin{array}{l}\text { Acquisition of information from other } \\
\text { platforms }\end{array}$ & $\begin{array}{l}\text { The app informs the user about the acquisition of information } \\
\text { from other platforms and about online and offline activities. }\end{array}$ \\
\hline & $\begin{array}{l}\text { Use of personal data (e.g., saving location } \\
\text { based-data over time; deleting past } \\
\text { location-based data) }\end{array}$ & $\begin{array}{l}\text { The app informs the user about how (and for how long) it will } \\
\text { use personal data. The user can access collected personal } \\
\text { data, erase location-based data, etc. }\end{array}$ \\
\hline \multirow[t]{5}{*}{ Model } & Name or type of the model & $\begin{array}{l}\text { Specific reference to the current algorithm version, } \\
\text { explanation of the differences between two different releases. }\end{array}$ \\
\hline & Input variables selection & $\begin{array}{l}\text { The main input variables the user can select to perform a } \\
\text { query (e.g., position on the map; metadata, etc.). }\end{array}$ \\
\hline & Metadata details & $\begin{array}{l}\text { Categorisation, provenance (e.g., public or private; editorial } \\
\text { or automatically generated content). }\end{array}$ \\
\hline & Additional details about the score & $\begin{array}{l}\text { Recentness of the score and frequency of the update } \\
\text { release. }\end{array}$ \\
\hline & $\begin{array}{l}\text { Identification of the user location on the } \\
\text { map }\end{array}$ & $\begin{array}{l}\text { The app informs the user about the criteria it uses to } \\
\text { determine his/her current location. }\end{array}$ \\
\hline \multirow[t]{4}{*}{ Inference } & Ranking & Criteria determining the (organic) ranking. \\
\hline & Score of a POI & Factors determining the score of a POI. \\
\hline & Personalisation & $\begin{array}{l}\text { Personalised (organic) results and (hidden) algorithmic } \\
\text { suggestions as a result of user activity. }\end{array}$ \\
\hline & Personalisation settings & $\begin{array}{l}\text { The user can set personalised preferences and filters for } \\
\text { organic results. }\end{array}$ \\
\hline
\end{tabular}




\begin{tabular}{|c|c|c|}
\hline & Paid placement & $\begin{array}{l}\text { Resources appear as "sponsored content" on top or } \\
\text { alongside the search results. }\end{array}$ \\
\hline & Paid placement settings & The user can set personalised preferences for tailored ads. \\
\hline \multirow[t]{6}{*}{ Interface } & Information findability & $\begin{array}{l}\text { Ease of finding information on the app (e.g., how many steps } \\
\text { the user has to follow to find it. }\end{array}$ \\
\hline & Priority of information & Which information is prioritised in the user interface. \\
\hline & Visual relevance on the map & $\begin{array}{l}\text { Criteria determining which POls are more relevant than } \\
\text { others on the map. }\end{array}$ \\
\hline & Extra features for premium accounts & Disclosure of extra features for premium accounts. \\
\hline & Editability of UGC & $\begin{array}{l}\text { The user can upload, edit or comment on location-based } \\
\text { information (e.g., photo, review). }\end{array}$ \\
\hline & Attribution of UGC & The author/editor of each content is revealed. \\
\hline
\end{tabular}

Table 1 - Model for analysing the algorithmic transparency of location-based platforms

With reference to the Diakopoulos and Koliska model, the four layers were interpreted as a continuum, given that they all mutually influence each other. For analytical purposes a distinction was made between the Model and the Inference layers. The Model layer includes the input variables used by proprietary algorithms to describe, classify or associate a POI. The Inference layer focuses more on the output side and aims to rank different resources. It includes the ranking of POIs in the search results page; the use of personalisation to infer a particular user's intention; the use of sponsored ads along or on top of the search results. As a result, the Inference layer closely cooperates with the Interface layer, thus affecting the overall visibility of the POIs on the user side.

As mentioned above, several official documents were consulted (privacy policy, terms of service, data policy, guidelines, tutorials and help pages). The analysis also took into consideration the communication style and the clarity of information for different target groups. We firstly analysed documents given to users during their initial use of the app and then, to find information addressing algorithm transparency, there was an intentional search within the mobile app and even within the official website, if needed. Internal links in official documents were also analysed. In relation to the three mentioned platforms, it was easier to find information on Tripadvisor, considering it is a thematic service and documents are hosted on the platform itself. On the other hand, it was frequently necessary on Google Maps and Instagram to perform several queries in order to find what we were looking for was being searched for. Indeed, the platforms are owned respectively by Google and Facebook, therefore official documents are hosted on the main company website. Furthermore, official documents (e.g., privacy policy, terms of service, data policy) address all services offered by the company. For example, in order to find information about a location-based platform (e.g., Instagram), users have to scroll through all Facebook documents.

\section{Results and discussion}

\subsection{Data}

In the context of location-based platforms, the availability of specific data shapes the data-processing phase. Indeed, the algorithmic model transforms the data and provides personalised results for different types of user. In relation to the aforementioned model (table 1), the most interesting factors emerging from the data layer are: 1) the acquisition of information from other platforms; 2 ) the use of personal data (e.g., saving location based-data over time; deleting past location-based data). 
Location-based platforms acquire additional information from third parties about a user's online and offline activities. Subsequently, these data are used to personalise the user experience and provide tailored ads. For example, Google Maps and Instagram collect information from other apps being used by the user. Google does not provide the whole list of partners, while Instagram does. Interestingly enough, Instagram, owned by Facebook, automatically receives information in real-time from Facebook about a user's online and offline activities, for instance, activities that the user has carried out outside of Facebook, such as websites visited, purchases made, etc.

In relation to transparency, another interesting issue is the use of personal data by the above mentioned platforms, with a specific interest in the use of location-based information in data processing. The analysis on the official documentation released by the platforms, whether accessed in the registration phase or via the platform settings, reveals that platforms do not provide sufficiently clear explanations about the processing of personal data. Furthermore, most of the time, even when they provide this information in the registration phase, they do not always offer 'granular' consent (i.e., users can opt into only some type of data processing but not others and do not have any leeway). As a result, users are initially forced to read very long privacy policies and accept the whole terms of service, data policy, and privacy policies, postponing their eventual opt-out only in relation to some issues (e.g., deactivating the saving of some data; deleting data already collected by the platform). Documents officially released by the platforms explicitly divulge only some information about the saving, use and deletion of personal data. Moreover, an interesting distinction emerges between storage options offered to suppliers and consumers. Indeed, suppliers can select for how much time they want to store their data; for example, on Google Maps, suppliers using Google Analytics can choose among different options (the minimum storage duration is no less than 14 months). Google Maps' consumers can set for how long they want to store their activity (Web \& App activity settings) and can manage the deletion of only certain types of data that the app automatically saves. However, Google does not explain which types of data they refer to. Instagram (and Facebook) provides information about how it automatically saves and stores consumer data. The user has to explicitly ask Instagram to delete personal data, otherwise data are stored without any time limit, unless the user deletes the account.

Another interesting topic is related to the transparency concerning the saving and deletion of location-based data. References to the settings of location-based data are mentioned in the main policy documents. A basic example of location-based data is information related to the location of photos published on social media. While several users intentionally share their location-based data via check-ins, tagging themselves into a location or publishing a review, platforms also infer some 'hidden' data about location from meta-data attached to multimedia content (picture, video, etc.) and, as mentioned below, automatically use them to provide more personalised services. For example, Instagram acquires location from event attendance, infers location by analysing user Internet connection and may even access the device's precise position even when the app is not being used. A very different level of transparency was observed in relation to the saving of user location: Tripadvisor does not provide any options, while Instagram allows consumers to disable the saving of user location ("Location Services" settings). Google Maps offers several options; however, only skilled users can personalise location-based data settings. Indeed, to delete location-based data the consumer has to carefully read official documents (such as the Google Maps Help, 2019) and then follow a two-step procedure that involves disabling the "Google location History" option and then the "Web \& App Activity", which also saves and stores the user's positions. Therefore, the deletion of 
location-based data is not effective with only the selection of the most intuitive option ("Google location History" deactivation) and requires an additional action ("Web \& App Activity" deactivation) that the consumer can only become aware of having read official policy documents.

\subsection{Model}

Location-based platforms adopt different assumptions and rules to process the infinite availability of information. The Model layer includes the input variables used to describe, classify or associate a POI and then used to perform a query, such as keywords, hashtags or locations (i.e., neighbourhood, cities, regions, etc). The focus of this study is on the algorithmic transparency of these variables.

Metadata (i.e., a set of data that describes other data) are an interesting example of the input options users can use to perform a query (consumer side) or manage their activity (supplier side). Metadata are general information about a POI, such as its main features and categories. Categories are tags for food and drink facilities describing, for example, the price range, the type of cuisine (European, Mexican, Neapolitan, Wine bar), etc. The variety of these categories is not a neutral choice and orients the classification of a place toward a specific cultural model. For example, a Chinese restaurant can be classified as a generic ethnic restaurant, thus providing a very Eurocentric vision of the world, or as a Cantonese restaurant, i.e., a specific Chinese regional cuisine. The platforms selected for this study do not provide detailed information about either the origin of these metadata (e.g., editorial generated; user generated; automatically generated content) or about the procedure for creating it. Moreover, some other categories are ambiguous, such as "Good for" in Tripadvisor.

Interestingly enough, the first distinction between the information provided by platforms to consumers and suppliers is that the latter are given more details about the whole procedure for the creation of these categories. On Tripadvisor, for instance, suppliers are informed that the categories are predetermined by the platform and that they can select a category to classify their venue (e.g., type of cuisine). Moreover, Tripadvisor explains the factors and criteria determining the attribution of specific categories. In relation to the "Best Value", Tripadvisor generically explains the meaning of this metadata only to suppliers: "Properties ranked using traveller ratings, confirmed availability from our partners, prices, booking popularity, location and personal user preferences" (Tripadvisor, 2019). Moreover, Google Maps explains only to suppliers how it infers metadata about POIs (e.g., review summary, popular times, etc.), while Instagram does not explain at all how it determines metadata regarding POIs (e.g., address, price, opening hours, etc.). Furthermore, the criteria adopted for the description or preview of a venue are vaguely defined. For example, Google Maps "review snippets" are defined as follows: "Review snippets are algorithmically selected quotes from Google users that provide information on the keywords that are most mentioned by reviewers" (Google My Business, 2019). Another example of an input variable is the user's location. Each platform uses several methods to infer the user's location: they mainly rely on the GPS, location of nearby Wi-Fi or connection to a cell-tower. Most of the time the user's location information is clearly intelligible, sometimes problems may arise if the user is in a disputed territory or in relation to a contested border.

Moreover, none of the platforms make any specific reference in their official documents to the name or the type of model they use to describe, classify or associate a POI, neither about the evolution of the model or about the differences between two different releases.

4.3. Inference 
The Inference layer focuses on the transparency of the output side. We focused on three main issues: 1) the ranking of a POI in the organic search results; 2) how the algorithm infers a particular user's intention and then suggests specific content (personalisation and relative settings); 3 ) sponsored ads and relative settings. On location-based apps the overall organic ranking of venues greatly influences their visibility and, therefore, the chance of getting new customers. When performing location-based queries, platforms determine the overall ranking according to several criteria: the score of the single POI among others. On Tripadvisor, for example, the score of a POI is expressed through a 'bubble rating' (with one bubble meaning 'terrible' and five bubbles meaning 'excellent') and "takes into account the quality, quantity, and age of individual travellers' ratings and reviews" (Tripadvisor Help Center, 2019). On Google Maps the score of a POI is expressed through a five-star scale. Instagram does not rate venues. Google and Tripadvisor do not inform users about the weight of the above mentioned factors, how recent the current score is or about how frequently scores are released. Subsequently, the overall organic ranking for a location-based query (e.g., restaurant) is influenced by the score of single POIs and a mix of proprietary criteria. For example, on Tripadvisor the "Popularity Index rank" "compares businesses and other places of interest to travellers based on their popularity, as measured by the quality, quantity, and recency of their content on TripAdvisor" (Tripadvisor Help Center, 2019). According to Tripadvisor "the Popularity Index is normally updated once a week". As Figure 1 shows, this means that a venue with 4.5 bubbles and more than 10,0oo reviews gets a better ranking than a venue with 5 bubbles and only 100 reviews.

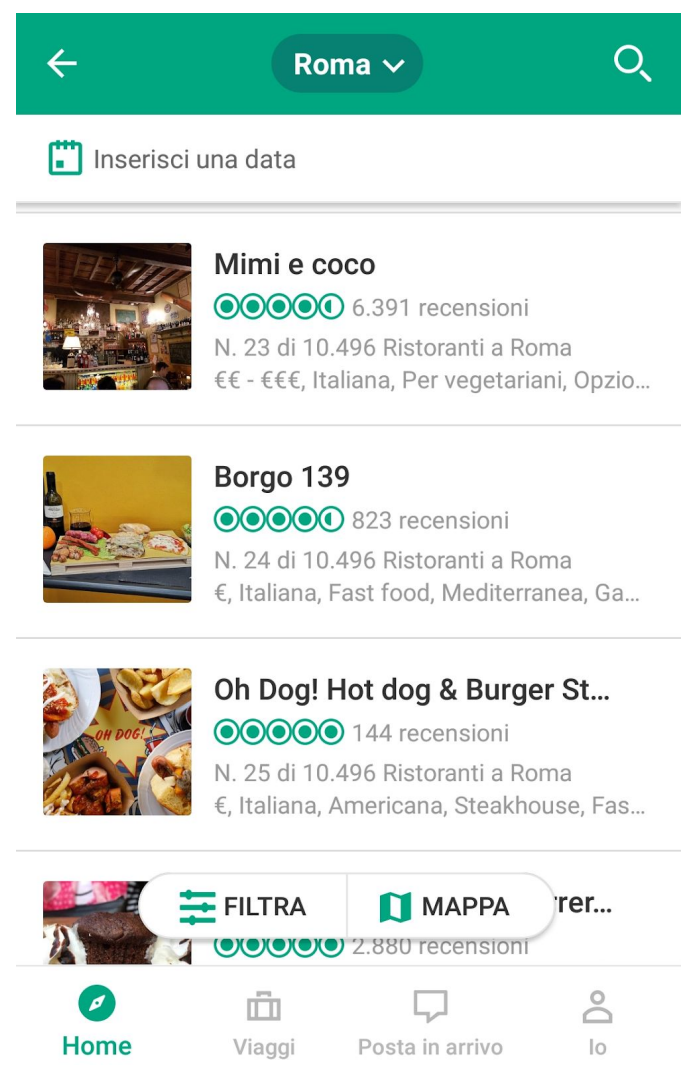

Figure 1 - Organic ranking on Tripadvisor. Screenshot taken by the authors.

In relation to personalisation, it is possible to distinguish between two different processes: 1 ) the personalisation of (organic) results that come from preferences intentionally set by the consumers; 2) 'algorithmic suggestion', a personalisation that builds on individual user activity and aggregated trends. Indeed, in order to maximise clicks, location-based algorithms - and digital algorithms in general - personalise search results according to several activities performed online 
(e.g., previous queries) and offline (e.g., places visited). Apps are not transparent about this hidden personalisation and do not reveal to the consumers when and how algorithms presume a particular user's intention or interest and arrange search results accordingly. For example, a user usually searches for Greek food, therefore the app will presume he likes Greek cuisine and will prioritise information about Greek restaurants. This results in a news feed that automatically personalises organic information without giving any feedback about which criteria are at play.

Advertising is the main source of revenue for these platforms. On Tripadvisor, Google Maps and Instagram, suppliers can get a prominent positioning in return for a placement fee (i.e., paid placement): sponsored content is marked as "sponsored ads" (with a specific icon) and appear on the top or alongside organic search results. Companies provide information, tutorials and guidelines to suppliers to explain how sponsored ads work and how suppliers can set advertising campaigns to reach the intended target (e.g., suppliers can use the 'Smart Ad' on Google Maps to target geographic area searches for phrases related to their business). Google Maps privacy policy provides general information to users about the information collected for personalising ads (e.g., cookies or user activity); Instagram Data Policy explains that the app uses "interests, actions and connections" about the user to personalise ads and sponsored content. However, the three apps do not give information about the criteria determining the visualisation of single ads. On Google users can manage ad personalisation; however, interestingly enough, even when personalisation is turned off "ads can still be targeted with info like your general location or the content of the website you're visiting" (Google Ads Help, 2019).

\subsection{Interface}

The design of an interface communicates to the user the most important functions of a platform and reflects the intentions of the designers. The analysis conducted here revolves around two main factors: the visual relevance of POIs on the map; the disclosure of extra features for premium accounts.

Location-based apps visualise places of interest on a map. For this analysis, several queries were performed on the selected platforms and several issues were noticed related to interaction with the map. Indeed, on Tripadvisor and Google we found one major transparency issue: the visual relevance of location-based data on the map and the transparency of the criteria determining it. An interesting example of the visual relevance of location-based data is the visualisation of different markers on the maps. Indeed, on Tripadvisor and Google Maps, the user can visualise search results directly on the map, and each venue is marked as a red dot on it. Due to the limited space on the map, marker density is displayed differently at different levels of zoom: upon opening the app, only few elements appear on the map (Figure 2), then, when zooming in, the map gets more and more detailed and new venues pop-up (Figure 3.1 and Figure 3.2). As a result, some names of POIs appear immediately at the first level of zoom and others appear only(?) when zooming in. Interestingly enough, on Google Maps some venues appear on the map even before the user performs a query (Figure 2). The visual relevance on the map plays an important role because it influences the findability of different places and their overall visibility; however, as far as we know, users (consumers and suppliers) are not informed about the criteria determining how venues are displayed at different levels of zoom. It can be supposed that some of the criteria that are at play might include: commercial interests (e.g., premium accounts get immediate visibility at the first level of zoom); popularity of the venue (e.g., more popular venues get better visibility); user interests and habits. 

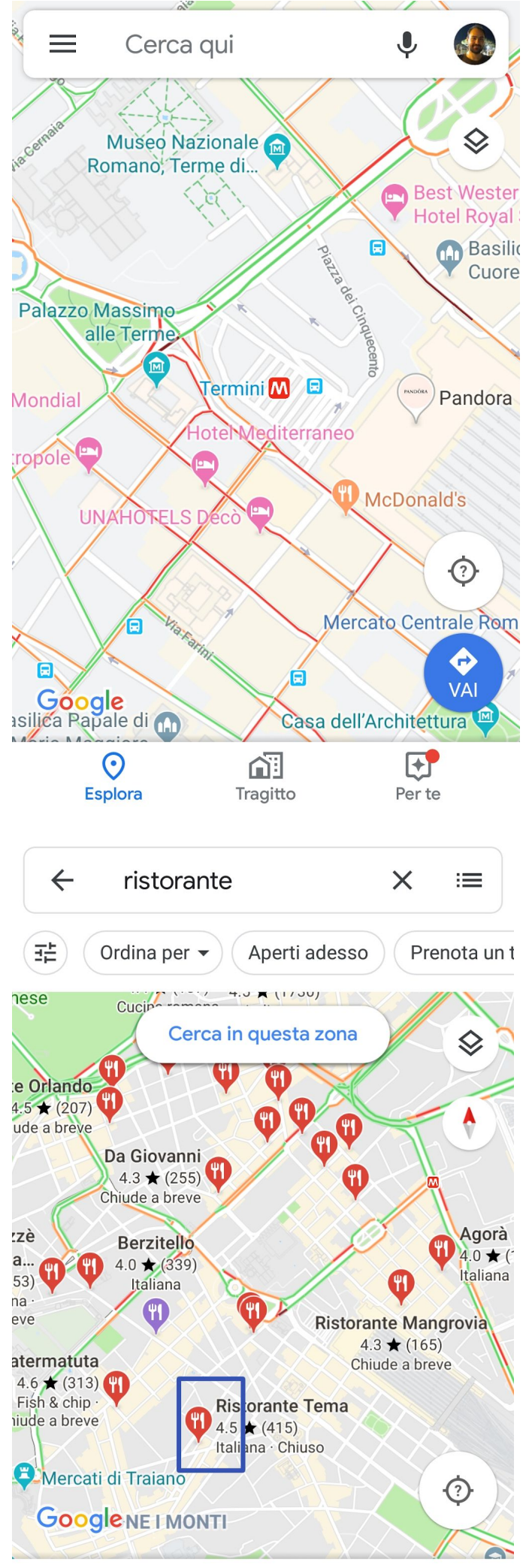

Mostra elenco
Figure 2 - Google Maps interface at zoom level zero (Rome, Italy, Termini area) before entering a query. Screenshot taken by the authors.

Figure 3.1 - Google Maps interface when zooming in for the first time (Rome, Italy, Termini area). Search results for "Ristorante" (restaurant). The selected area in the blue box is the one shown in 


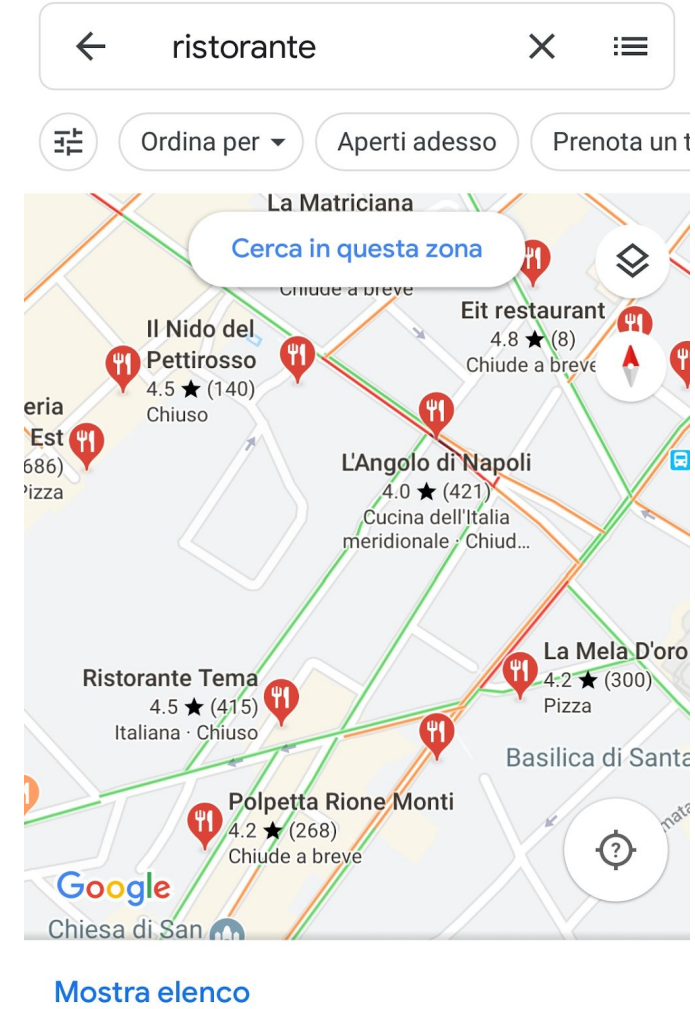

Figure 3.2. Screenshot taken by the authors.

The factor "disclosure of extra features for premium account" describes the opportunity for suppliers with a premium account to personalise their profile, thus getting greater visibility. Tripadvisor suppliers can pay to enrich and personalise their profiles through different features that are not available for basic account holders. For example, they can create a slideshow of selected photos, choose the photo order and highlight a favourite review. The consumer is informed that these improvements are only granted to premium accounts (monthly subscription: 9o euro), except for the slideshow. In this latter case, the user does not know that the supplier is paying for a more attractive layout and may believe the venue is innately more attractive as a result. Indeed, we discovered the existence of the slideshow option in this analysis only by looking at the documentation provided to suppliers. Google Maps does not allow premium accounts to embellish or enhance their profiles.

\section{Conclusion}

In accordance with Rader, Cotter and Cho (2018), it was expected that location-based platforms would be less transparent about the data they manage and model they use and slightly more transparent about the inferences (suggestions and recommendations) they propose. The analysis confirms this hypothesis. As Obar and Oeldorf-Hirsch (2018) explained, users tend to ignore privacy policy and terms of service, especially when the text is very long. Location-based apps (Tripadvisor, Google Maps and Instagram) provide a very long list of the data they collect and how they use them; however, they do not always offer 'granular' consent, that is, users are forced to accept such documents, postponing their eventual opt out only in relation to some issues (e.g., deactivate the saving of some data; delete data already collected by the platform, etc.). As a result, users experience a pseudo-transparency: they must initially accept as a whole the privacy policy, terms of service and data policy and cannot modify the exploitation of their personal data. The model layer is the most invisible layer and, therefore, is very opaque. Criteria used to describe, classify and associate a place (metadata) are presented as objective and neutral; however, they are affected by specific cultural 
assumptions, ordering models and commercial strategies, thus greatly influencing the user's overall experience of of places.

As expected, the inference layer is more transparent, mainly toward suppliers, given that business interests favour the disclosure of information. Indeed, suggestions and recommendations toward consumers greatly influence the visibility and popularity of a venue; moreover, knowing how the system works can convince a supplier to subscribe to a premium account and invest their budget in advertising. More specifically, platform owners provide information to suppliers in relation to the criteria determining: the ranking of a POI in organic search results; how sponsored ads work; how suppliers can set advertising campaigns. Furthermore, on Tripadvisor, suppliers with a premium account can enhance their profile, thus getting better visibility (e.g., slideshow, selection of the best review, etc.). Interestingly enough, location-based apps are not transparent toward consumers in relation to algorithmic personalisation presuming a particular customer's intention and thus determining inferences. This is the case of so-called 'algorithmic suggestions' where information matching presumed user interests is prioritised in order to maximise clicks.

The analysis reveals how platform documents communicate transparency differently toward suppliers and consumers. As expected, it was observed that location-based platforms are more transparent toward suppliers than consumers. Information is disclosed to suppliers regarding mainly the ranking of organic search results and sponsored ads. Interestingly enough, most of the tools to simplify information, such as guidelines (e.g., Google My Business, Tripadvisor Insights), tutorials and help pages, are oriented toward suppliers to satisfy a business interest. There was no indication in the analysis of a similar attention toward consumers, in relation to several factors. For example, platforms do not provide information about the criteria determining several transparency factors, such as: the massive use of data from third parties; the meaning of metadata; how different POI are displayed at different levels of zoom on the map.

This trend confirms that the commercial interests of location-based platforms favour the algorithmic transparency and visibility of location-based content. Indeed, the main aim of these platforms is to acquire a larger amount of user data in order to capture the so-called 'behavioural surplus' (Zuboff, 2019) and generate more profit later. Moreover, the intelligence of the algorithms themselves depends on the amount of data accumulated and, for this reason, the platforms must collect as many users as possible. As a result, suppliers help platforms in reaching this aim, given that their interests match. The commercial logic of platform owners and suppliers is to increase the number of users; in this way platforms can feed the database with new users and suppliers can increase their customer base. As a result, this utilitarian motivation leads suppliers to accepting practices adopted by the so-called 'Surveillance Capitalists' (Zuboff, 2019).

In conclusion, this mechanism is also reflected in the differing transparency for users and suppliers. As a result, the commercially oriented ideology of the platforms determines the visibility of location-based content and prioritises venues adhering to their algorithmic logic, leaving behind venues that do not adopt the proposed marketing strategy.

\section{Further research steps}

This paper is part of a wider research project investigating the transparency of location-based algorithms. The same analytical model will be used to investigate user perceptions of the algorithmic transparency of location-based platforms. We will collect 'elicit' data from consumers and suppliers of the three location-based apps selected for this study (Tripadvisor, Google Maps and Instagram). 
Moreover, in relation to suppliers, there will be investigation into how they cope with official insights and reports in relation to their eventual use of premium accounts. The overall aim is to explore consumer and supplier understanding of the algorithmic transparency of location-based platforms, for example, if (and how) consumers believe the platform ideology shapes their spatial experience and how suppliers deal with the platform algorithmic logic assessing reputation, visibility, etc. In-depth interviews have already been carried out with suppliers (May 2019) and consumers.

Further research is also needed to explore the algorithmic imaginary (Bucher, 2017) of search engine marketing specialists (SEO/SEM) in relation to the transparency of location-based platforms. Indeed, there is intensive debate among experts on closed social media groups about the evolution of algorithmic models, comparing different releases and examining, almost in real time, marketing strategies used to increase the visibility and popularity of different venues. It is clear these experts play an important role in adopting (and maybe shaping) algorithmic culture and in popularising it for the general public.

\section{References}

Barabási, A. L. (2004). Link. La scienza delle reti. Torino: Einaudi.

Bucher, T. (2017). The algorithmic imaginary: Exploring the ordinary affects of Facebook algorithms. Information Communication and Society, 20(1), 30-44.

Burgess, J., \& Green, J. (2009). YouTube. John Wiley \& Sons.

Cardon, D. (2018). Che cosa sognano gli algoritmi. Milano: Mondadori.

Diakopoulos, N. (2015). Algorithmic accountability: Journalistic investigation of computational power structures. Digital Journalism, 3(3), 398-415.

Diakopoulos, N., \& Koliska, M. (2017). Algorithmic transparency in the news media. Digital Journalism, 5(7), 809-828.

Diakopoulos, N., Trielli, D., Stark, J., \& Mussenden, S. (2018). I Vote For-How Search Informs Our Choice of Candidate. Digital Dominance: The Power of Google, Amazon, Facebook, and Apple, M. Moore and D. Tambini (Eds.), 22.

Fuchs, C. (2017). Social media: A critical introduction. Sage.

General Data Protection Regulation (GDPR) (2019) https://eugdpr.org/ (Retrieved 31 January 2019)

Gillespie, T. (2017). Algorithmically recognizable: Santorum's Google problem, and Google’s Santorum problem. Information, Communication \& Society, 2o(1), 63-80.

Google Ads Help (2019), “Controllare quali annunci vengono mostrati” eng. Control the ads you see" available at

https://support.google.com/ads/answer/2662856?co=GENIE.Platform\%3DAndroid\&h $1=$ it 
Google Maps Help (2019), “Guida di Google Maps, available at https://support.google.com/maps/?hl=it\#topic=3092425

Google My Business (2019), "Types of business summaries on Google Maps", available at https://support.google.com/business/answer/6o88158?hl=en-GB

Goodman, B., \& Flaxman, S. (2017). European Union regulations on algorithmic decision-making and a "right to explanation. AI Magazine, 38(3), 50-57.

Granka, L. A. (2010). The politics of search: A decade retrospective. The Information Society, 26(5), 364-374.

Introna, L. D. (2016). The algorithmic choreography of the impressionable subject. In Algorithmic Cultures (pp. 38-63). Routledge.

Introna, L. D., \& Nissenbaum, H. (200o). Shaping the Web: Why the politics of search engines matters. The information society, 16(3), 169-185.

Jenkins, H., \& Deuze, M. (2008). Convergence culture. Sage.

Laidlaw, E. B. (2008). Private power, public interest: An examination of search engine accountability. International Journal of Law and Information Technology, $17(1)$, 113-145.

Law. J. (2009). Actor network theory and material semiotics. Social theory, 141.

Law, J., \& Hassard, J. (1999). Actor network theory and after, Blackwell Publishing.

Mayer-Schönberger, V., \& Cukier, K. (2013). Big data: A revolution that will transform how we live, work, and think. Houghton Mifflin Harcourt.

Nieborg, D. B., \& Poell, T. (2018). The platformization of cultural production: Theorizing the contingent cultural commodity. New media \& society, 20(11), 4275-4292.

Obar, J.A., \& Oeldorf-Hirsch A.. (2018). The biggest lie on the Internet: Ignoring the privacy policies and terms of service policies of social networking services. Information, Communication \& Society (2018), 1-20.

Pan, B., Hembrooke, H., Joachims, T., Lorigo, L., Gay, G., \& Granka, L. (2007). In google we trust: Users' decisions on rank, position, and relevance. Journal of computer-mediated communication, 12(3), 801-823.

Pariser, E. (2011). The filter bubble: What the Internet is hiding from you. Penguin UK.

Parisi L. (2015) 'Where 2.o'. Exploring the Place Experience of 'Hyperconnected' Digital Media, Users in Special Issue: Other Senses of Place: Sociospatial Practices in the Contemporary Media Environment, Sociologica, 03/2015, Il Mulino.

Pasquale, F. (2015). The black box society: The secret algorithms that control money and information. Harvard University Press. 
Pickering, A. (1995), The Mangle of Practice: Time, Agency and Science. Chicago, University of Chicago Press.

Rader, E., Cotter, K., \& Cho, J. (2018). Explanations as Mechanisms for Supporting Algorithmic Transparency. In Proceedings of the 2018 CHI Conference on Human Factors in Computing Systems (p. 103). ACM.

Salmons, J. E. (2015). Doing qualitative research online. Sage.

van Dijck, J., Poell, T., \& de Waal, M. (2018). The platform society: public values in a connective world. Oxford University Press.

Tripadvisor (2019), "6 Things You Should Be Doing to Drive More Bookings from TripAdvisor Search Results”, available at https://www.tripadvisor.com/TripAdvisorInsights/w3403

Tripadvisor Help Center (2019), "How does the Popularity Index differ from the overall bubble rating?"

https://www.tripadvisorsupport.com/hc/en-us/articles/200614027-How-does-the-Pop ularity-Index-differ-from-the-overall-bubble-rating

Varkaris, E., \& Neuhofer, B. (2017). The influence of social media on the consumers' hotel decision journey. In Journal of Hospitality and Tourism Technology 8(1), 101-118.

Zuboff, S. (2019). The Age of Surveillance Capitalism: The Fight for a Human Future at the New Frontier of Power: Barack Obama's Books of 2019. Profile Books. 


\section{Appendix A}

(documents consulted, as they appeared online on the 31 May 2019)

\section{Google}

Google Ads Help: https://support.google.com/google-ads\#topic=7456157

Google Maps Help: https://support.google.com/maps/?hl=it\#topic=3092425

Google My Business: https://support.google.com/business/answer/3038063?hl=it

Google Privacy and Terms: https://policies.google.com/

Tripadvisor:

Help Center: https://www.tripadvisorsupport.com/hc/it

Privacy Policy: https://tripadvisor.mediaroom.com/IT-privacy-policy

Terms of Use: https://tripadvisor.mediaroom.com/IT-terms-of-use

Instagram:

Data Policy: https://help.instagram.com/519522125107875

Terms of use: https://help.instagram.com/581066165581870

Platform Policy: https://www.instagram.com/about/legal/terms/api/ 